\title{
C-Reactive Protein-Guided Approach May Shorten Length of Antimicrobial Treatment of Culture-Proven Late-Onset Sepsis. An Intervention Study
}

\author{
Renato C. Couto, José A. A. Barbosa, Tânia M.G. Pedrosa and Fernando M. Biscione \\ Health Sciences and Tropical Medicine Post Graduate Course, Medicine High School, \\ Minas Gerais Federal University, Belo Horizonte, MG, Brazil
}

\begin{abstract}
Late-onset sepsis (LOS) (i.e., sepsis in a neonate after 72 hours of life) is associated with high mortality and significantly prolonged antibiotic exposure and hospital stay in neonates admitted to intensive care units (ICU). In this study, we assessed the reliability of serum C-reactive protein (CRP) as a determinant of antimicrobial treatment duration of LOS. From January 1996 to December 2002, all consecutive infants aged $\leq 28$ days admitted to a single medical-surgical ICU and diagnosed with primary LOS were enrolled in a prospective, intervention trial with historical controls. Only blood culture-positive LOSs were included. Exclusion criteria were: age $>28$ days at diagnosis of LOS, development of site-specific infection, and central venous catheter-related LOS. From January 1996 to July 1998 (historical control group), antimicrobial treatment of LOS was offered for at least 14 days. From August 1998 to December 2002 (intervention group), neonates underwent serial semiquantitative measurements of serum CRP, and antimicrobial treatment was discontinued when CRP was $\leq 12 \mathrm{mg} / \mathrm{L}$. Primary efficacy endpoint was the duration of antimicrobial therapy. Secondary efficacy endpoints were the proportion of relapsing sepsis within 72 hours of antibiotic withdrawal and the overall mortality rate. The historical control group comprised 76 neonates developing 85 episodes of LOS; 138 LOS occurring in 120 patients comprised the intervention group. Length of antimicrobial treatment of LOS was significantly shorter during the second study period (16 days vs. 9 days, $\mathbf{p}<\mathbf{0 . 0 0 1})$. Secondary efficacy endpoints showed similar rates of relapsing sepsis and overall mortality in both time periods.
\end{abstract}

Key-Words: C-reactive protein, newborn, neonatal intensive care units, sepsis, antibiotics.

Late-onset sepsis (LOS) (i.e., that occurs in a neonate after 72 hours of life) is associated with significantly prolonged hospital stay and mortality among newborns admitted to neonatal intensive care units (NICU) [1-3]. The incidence of LOS increases with both decreasing birthweight and gestational age, and has been reported as occurring in approximately $25 \%$ of very-low birthweight infants [2-5]. The pathogenesis and spectrum of organisms responsible for earlyonset sepsis differs from that associated with LOS [6], this pattern becoming apparent from day 2 of NICU admission onwards [4]. Prompt and adequate antibiotic treatment is known to dramatically impact on adverse outcomes. NICUinfants with suspected LOS are tipically treated with empiric broad-spectrum antibiotics until culture results are available. A number of guidelines exist on the appropriate use of antibiotics for this condition, raising awareness about the negative consequences of antimicrobial overuse [7]. Despite this, excessive and/or inappropriate antibiotic prescription continues to occur worldwide [2,5]. Currently, there is a lack of consensus among neonatologists on the most appropriate duration of antimicrobial treatment for suspected or cultureproven LOS [5]. Current literature recommends various treatment endpoints of 7-14 days for culture-positive neonatal infections [8]. Adjunctive laboratory data may assist the clinician in supporting the diagnosis of neonatal sepsis while Received on 10 November 2006; revised 23 March 2007.

Address for correspondence: Dr. Renato C. Couto. 190 Prof. Alfredo Balena avenue, suite 7003, Santa Efigênia, Belo Horizonte, Minas Gerais, Brazil. Zip-code: 31130-100. Phone: (0055) 31 3248-9640.

The Brazilian Journal of Infectious Diseases

2007;11(2):240-245. (C) 2007 by The Brazilian Journal of Infectious Diseases and Contexto Publishing. All rights reserved. awaiting for blood culture results, and in determining the optimal duration of antimicrobial therapy. Acute-phase reactants, such as procalcitonin, serum amyloid-A, C-reactive protein (CRP), tumor necrosis factor-á, interleukin (IL)-1, IL-6, IL-8, and IL-1 antagonist receptor, among others, have been advocated for such purposes $[9,10]$. In particular, a CRP level that returns to the normal range in a neonate diagnosed with sepsis may indicate that the duration of antibiotic treatment has been sufficient, allowing earlier discontinuation of antibiotics.

In our institution, until June 1998, our practice was to guide antimicrobial treatment duration of primary culture-positive LOS following fixed time courses of antibiotics. Since then, we introduced a clinical pathway for neonatal sepsis, which relied primarily on serum CRP as a supportive criterion for withdrawal of antibiotic therapy in neonates with primary culture-positive LOS. The aim of the present work was to assess the reliability of this approach.

\section{Materials and Methods}

Patients and Setting

From January 1996 to December 2002, all consecutive infants aged $\leq 28$ days admitted to Neocenter S/A and diagnosed with primary culture-positive LOS were eligible for enrollment in this unblinded, prospective intervention trial with historical controls. Newborns with the following criteria were excluded: age $>28$ days at the time of diagnosis of LOS, patients developing a site-specific infection (i.e., pneumonia, meningitis, etc.), and diagnosis of central venous catheterrelated LOS.

Neocenter S/A is a 20-bed, non-universitary, medicalsurgical, high-complexity NICU at Belo Horizonte, Brazil. This 
NICU provides care for critically ill newborns, extremely low birth-weight premature infants, neonates for pre/post operative management, and those with congenital anomalies that require close observation and/or intervention. Neocenter S/A admits approximately 400 neonates per year, and does not accept transports. The permanent attending health care staff ratio includes a technical nurse per 2 beds, a professional nurse, a neonatologist and a medical resident per 10 beds, and a respiratory therapist. The NICU offers conventional and high frequency ventilation, nitric oxide therapy for pulmonary hypertension, and incubators with capability for regulation of the temperature and humidity, among others, and in-unit surgical procedures such as extracorporeal membrane oxygenation, cardiac catheterization, etc. For this trial, written standards based on published guidelines on nosocomial infection control and prevention [11-13] were followed by the attending personnel, including routine rate reporting and continuous medical and nursing staff training on infection control and prevention practices.

In accordance with our institutional protocols, the same initial clinical pathway is applied to all neonates at NICU admission, irrespective of the clinical condition. This includes a plan of clinical assessment and care concerning thermoregulation, ventilation, circulation, metabolism and nutrition. All infants undergo complete physical examination, continuous electrocardiography and pulse oximetry, and either central or peripheral venous catheterization as needed. Blood samples are drawn for arterial gasometry, blood cultures, complete hemogram, and sodium, potassium, chlorine and glucose measurement. Other therapeutic or diagnostic interventions are performed as dictated by clinical judgment.

\section{Data Collection and Definitions}

The data reported in this study are part of an ongoing institutional nosocomial infection surveillance program, in which data collection is performed using standardized forms by 2 independent, well-trained investigators. Prospective, active surveillance methods and data source include daily review of all medical charts, microbiology reports, and pharmacy files. Joint revision of medical records and discussion with the attending physician using a nonsystematic approach is carried out whenever discrepant data are reported by the 2 independent researchers.

Primary LOS was defined as development of sepsis not related to infection at another body site after 72 hours of life. The set of criteria for laboratory-confirmed bloodstream infection for patients $\leq 12$ months of age proposed by the Centers for Disease Control and Prevention in 1988 was adopted as case definition of sepsis [14]. Only culture-proven (i.e., blood culture-positive) sepses were included in the study. Blood cultures positive for organisms generally considered to be contaminants (e.g., Corynebacterium sp., Bacillus sp., micrococci, diphteroids, etc.) were excluded from analysis. Coagulase-negative staphylococci were considered true pathogens when 2 blood cultures drawn on separate occasions were positive and the organism was not related to infection at another site. For all other pathogens, microorganisms recovered at least in one blood culture were considered causative agents.

\section{Intervention}

From January 1996 to July 1998 (historical control group), antimicrobial treatment of culture-proven primary LOS was offered to all neonates for at least 14 days, and until complete clinical resolution was achieved. From August 1998 to December 2002 (intervention group), neonates diagnosed with LOS underwent serial semiquantitative measurements of serum CRP, and antimicrobial treatment was discontinued as soon as CRP serum levels fell below or were equal to $12 \mathrm{mg} / \mathrm{L}$, provided that all signs and/or symptoms of sepsis had subsided. CRP serum levels were measured at the diagnosis of LOS and every 48 hours thereafter, by means of a semiquantitative latex agglutination assay. Total white blood cell, immature-total neutrophil ratio and absolute neutrophil count did not determine duration of antibiotic therapy.

For both groups of infants, broad-spectrum antibiotics were empirically started at the time of suspicion of LOS, based upon the predominant antimicrobial resistance pattern at our institution. The choice of the empiric therapy was left at the discretion of the attending neonatologist, but was adjusted as necessary as soon as antimicrobial susceptibility tests became available. Bacterial strain biochemical identification was performed using non-authomated methods, and the agar disc diffusion test (Kirby-Bauer), using 1993, 1997 and 2000 NCCLS performance standards (and information supplements), was used for antimicrobial susceptibility testing.

\section{Data Analysis}

The primary efficacy endpoint for this study was the duration of antimicrobial therapy for LOS in both the historical control group and the intervention group. Length of antimicrobial treatment was recorded in days until hospital discharge or death. The secondary efficacy endpoint was the proportion of neonates with relapsing sepsis, defined as the development of either laboratory-confirmed bloodstream infection or clinical sepsis, according to CDC's 1988 definition criteria for patients $\leq 12$ months of age [14], within 72 hours of antibiotic withdrawal. An additional secondary efficacy endpoint was the overall mortality rate, as the direct atributable mortality due to sepsis is not always easy to determine in this critically ill population.

The chi-square test with Yates' correction or the Fisher's exact test (when any expected value in a 2x2 contingency table was lower than 5) were used for comparison of categorical data. Continuous, non-normally-distributed data were compared by means of Mann-Whitney's U test. For all statistics, the level of significance was set at $5 \%$ (i.e., $\alpha=0.05$, two-tailed). Epi Info for Windows, version 3.3.2 was used for statistical analysis. 
The study was approved by the Neocenter S/A's independent ethics committee and by the Federal University of Minas Gerais’ Institutional Review Board.

\section{Results}

From January 1996 to July 1998, 871 infants aged $\leq 28$ days were admitted to the NICU and 88 laboratory-confirmed primary LOS were recorded, for a LOS rate of $10.1 \%$. Three episodes of LOS met exclusion criteria, leaving 85 episodes developed in 76 infants for analysis. From August 1998 to December 2002, 1,264 newborns were admitted to the NICU and 200 primary culture-positive LOS were diagnosed, for a LOS rate of 15.8\%. Sixty-two episodes of LOS met exclusion criteria and were excluded from analysis, so 138 episodes occurring in 120 patients ultimately entered the study. The baseline characteristics of the cohorts are summarized in Table 1. The intervention group was characterized by lower gestational age, lower birthweight, and longer length of NICU stay as compared to the historical control group. All neonates diagnosed with LOS had a 48 hour serum CRP $>12 \mathrm{mg} / \mathrm{L}$ (data not shown). Overall, 227 microorganisms were isolated in 223 episodes of primary blood culture-positive LOS, 87 in the control group and 140 in the intervention group. Two neonates in each cohort had an episode of sepsis in which 2 pathogens were concomitantly isolated. Pathogen distribution is depicted in Table 2.

Results of the efficacy analysis are shown in Table 3. As can be noted, length of antimicrobial treatment for LOS was significantly shorter during the second study period (16 days vs. 9 days, $\mathrm{p}<0.001$ ). In a simple stratified analysis according to gestational age and birthweight, significant statistical differences towards a shorter duration of antimicrobial usage in the intervention group remained for all categories, except for those neonates aged $\geq 37$ weeks and those weighting $>2,500$ grams at birth, for whom a tendency, though not reaching statistical significance, was observed. Conversely, secondary efficacy endpoints showed similar rates of relapsing sepsis and overall mortality in both time periods (Table 3).

\section{Discussion}

Prolonged antimicrobial treatment is known to increase the likelihood of colonisation with antimicrobial-resistant organisms in neonates admitted to a NICU [7], so defining the

Table 1. Characteristics of study sample

\begin{tabular}{lccr}
\hline Variable & $\begin{array}{c}\text { Control group } \\
(\mathbf{n}=\mathbf{7 6})\end{array}$ & $\begin{array}{c}\text { Intervention group } \\
(\mathbf{n = 1 2 0 )}\end{array}$ & p-value ${ }^{-123}$ \\
\hline Gestational age (weeks) [median (range)]* & $32(24-40)$ & $30(23-38)$ & 0.002 \\
Birthweight (grams) [median (range)] & $1,322(580-4,160)$ & $1,220(430-4,000)$ & 0.034 \\
Length of NICU stay (days) [median (range)] & $37(0-243)$ & $43.5(6-388)$ & 0.047 \\
NICU: neonatal intensive care unit. & & & \\
\hline
\end{tabular}

*Data missing for 5 patients in the control group and 7 patients in the intervention group; $†$ Mann-Whitney’s U test.

Table 2. Causative agents in neonates with late-onset primary sepsis

\begin{tabular}{lccr}
\hline Isolated pathogen & $\begin{array}{c}\text { Control group } \\
\text { (n, \%) }\end{array}$ & $\begin{array}{c}\text { Intervention group } \\
\text { (n, \%) }\end{array}$ & p-value \\
\hline Gram-negative organisms & $65(75.5)$ & $86(63.3)$ & $0.076 \dagger$ \\
Klebsiella sp. & $34(39.5)$ & $66(48.5)$ & $0.24 \dagger$ \\
Pseudomonas sp. & $9(10.5)$ & $8(5.9)$ & $0.32 \dagger$ \\
Serratia sp. & $7(8.1)$ & $6(4.4)$ & $0.39 \dagger$ \\
Enterobacter sp. & $7(8.1)$ & 0 & $0.001 \ddagger$ \\
Alcaligenes sp. & $4(4.6)$ & 0 & $0.02 \ddagger$ \\
E. coli & $3(3.5)$ & $5(3.7)$ & $1 \neq$ \\
P. mirabilis & $1(1.2)$ & $1(0.7)$ & $1 \ddagger$ \\
Gram-positive organisms & $12(14)$ & $41(30.1)$ & $0.009 \dagger$ \\
S. aureus & $6(7)$ & $1(0.7)$ & $0.01 \ddagger$ \\
S. epidermidis & $6(7)$ & $34(25)$ & $0.001 \dagger$ \\
Enterococcus sp. & 0 & $6(4.4)$ & $0.08 \ddagger$ \\
Fungi & $9(10.5)$ & $9(6.6)$ & $0.44 \dagger$ \\
Candida sp. & $9(10.5)$ & $9(6.6)$ & $0.44 \dagger$ \\
Total* & $86(100)$ & $136(100)$ & \\
\hline
\end{tabular}

*Data missing for 1 patient in the control group and 4 patients in the intervention group; †Chi-square test; $\ddagger$ Fisher’s exact test. 
Table 3. Primary and secondary efficacy endpoints

\begin{tabular}{|c|c|c|c|}
\hline & $\begin{array}{c}\text { Control group } \\
(\mathbf{n}=\mathbf{8 5})\end{array}$ & $\begin{array}{l}\text { Intervention group } \\
\quad(\mathrm{n}=138)\end{array}$ & p-value \\
\hline \multicolumn{4}{|l|}{ Length of antimicrobial treatment } \\
\hline Pooled value (days) [median]* & $16(n=84)$ & $9(n=136)$ & $<0.001 \S$ \\
\hline$<37$ weeks GA (days) [median]† & $16(n=64)$ & $9(\mathrm{n}=117)$ & $<0.001 \S$ \\
\hline$\geq 37$ weeks GA (days) [median]† & $18.5(n=14)$ & $10(n=10)$ & $0.064 \S$ \\
\hline$<1,500$ grams (days) [median]‡ & $16(n=56)$ & $9(n=89)$ & $<0.001 \S$ \\
\hline $1,501-2,500$ grams (days) [median] $\ddagger$ & $17(n=9)$ & $8(n=34)$ & $0.046 \S$ \\
\hline >2,500 grams (days) [median] $\ddagger$ & $16(n=19)$ & $11(n=13)$ & $0.65 \S$ \\
\hline $\begin{array}{l}\text { Relapsing sepsis } \\
\text { No. (\%) }\end{array}$ & $2(2.4)$ & $2(1.4)$ & 0.64 \\
\hline \multicolumn{4}{|l|}{ Overall mortality } \\
\hline No. (\%) & 15 (17.6) & $22(15.9)$ & $0.88 \pi$ \\
\hline
\end{tabular}

GA=gestational age. *Data missing for 1 neonate in the control group and 2 neonates in the intervention group; †Data missing for 7 neonates in the control group and 11 neonates in the intervention group; łdata missing for 1 neonate in the control group and 2 neonates in the intervention group; §Mann-Whitney’s U test; 'Fisher’s exact test; ๆChi-square test.

optimal duration of antimicrobial usage for both prophylactic and therapeutic purposes is likely to be a primary strategy for the reduction of emergence and spread of resistant bacteria in the hospital environment [15].

Acute-phase reactants are produced and released when inflammation from any cause is present [10]. CRP is an acutephase protein synthesized in the liver in response to inflammatory cytokines [16]. It is a well-known classic pathway complement activator, as well as an opsonin, and plays a major role in innate immunity [16-18]. In healthy, vaginally-delivered babies, serum levels increase exponentially after birth, reaching a median peak concentration of about $5.6 \mathrm{mg} / \mathrm{L}$ within 48 hours [19]. Serum CRP peak values in neonates born by cesarean section are smaller and demonstrate delayed increases compared with those of babies born by vaginal delivery (median peak value of about $3.1 \mathrm{mg} / \mathrm{L}$ within 60 hours of birth) [19]. Lower birth weight ( $<2,500$ gr), but not lower gestational age ( $\leq 37$ weeks) is also associated with smaller CRP increases [19].

CRP serum levels may increase as much as 1,000-fold during an acute-phase response triggered by inflammation and/or tissue injury of both infectious and non-infectious sources, including neonatal sepsis [16,20-22]. Serum levels begin to rise within few hours of the onset of signs of infection or tissue injury, but this rise may lag $12-24$ hours $[16,21,23]$. There is generally a delay of up to 24-48 hours until peak levels are reached $[16,24]$. Serum CRP levels remain high as long as the injurious stimulus persists but, due to its short half-life (aproximatelly 20 hours), they fall rapidly after elimination of the (often microbial) source of inflammation $[16,24,25]$. During the neonatal period, an established upper normal CRP level of $10 \mathrm{mg} / \mathrm{L}$ has been identified in most studies as indicating the presence of sepsis or infection $[8,21,23,26-$ 28], but other authors have used different upper normal reference levels $[21,29,30]$. Conflicting values have added much to the confusion and uncertainty to the use of CRP in clinical practice. The decision to use the cutoff level of $12 \mathrm{mg} /$ $\mathrm{L}$ in our study resulted from the semiquantitative nature of the latex agglutination assay available at our institution at the time of the study.

A single initial determination of serum CRP at the onset of illness is poorly sensitive for diagnosing bacterial infection in neonates, but the sensitivity can be enhanced by serial determinations within 12-24 hours [21,26,31]. Serial determinations are also highly reliable for the diagnosis of LOS, with sensitivity of up to $97.5 \%$ and negative predictive value of up to $98.8 \%$ [23,27]. Conversely, the positive predictive value of a raised CRP for infection is poor [23,27]. False positives in NICU-admitted neonates may arise from intraventricular haemorrhage, meconium aspiration, necrotising enterocolitis, perinatal asphyxia, pneumothorax, surgery and immunisation [16,21].

The high negative predictive value of normal CRP serum levels for bacterial infection in neonates makes it an attractive alternative for monitoring disease activity and patient response to antimicrobial therapy over time, and for guiding the duration of antibiotic therapy after a primary diagnosis of invasive infectious disease has been made $[10,28,32]$. In our intervention study, the application of a strategy of serial measurement of serum CRP until levels fell below a fixed threshold in neonates with culture-proven primary LOS allowed us to safely reduce overall antimicrobial usage as compared to historical controls. This reduction was statistically significant across birthweight and gestational age categories, except for those neonates aged $\geq 37$ weeks and those weighing $>2,500$ grams at birth. The tendency observed for these latter categories suggests that the lack of statistical significance is likely to be the result of a low statistical power owing to the small sample size of these categories. Interestingly, this CRPguided approach could be applied with no evidence of increased adverse outcomes, as measured by overall mortality and sepsis relapse due to treatment failure. 
The results suggested by our study are in line with previous reports $[8,28]$, although differences in study design and case definition make generalizations impossible. We are unaware of any published study using such a stringent criterion for case definition as our (i.e., only culture-proven primary LOS) for the hole sample of neonates. We believe that the definition we adopted strengthens the conclusions as compared with previous reports. Although we acknowledge that using blood culture results for the diagnosis of neonatal sepsis may have several drawbacks [30], we are confident that we adopted a case definition that accurately distinguishes culture contaminants from true systemic infections.

The results reported in this study must be interpreted with much caution. It has been suggested that perinatal administration of antibiotics to reduce the risk of group B streptococcal (GBS) disease may influence the outcome and pathogen distribution of early-onset sepsis [33]. This topic is of great importance since guidelines for the prevention of perinatal GBS disease became available in the early $1996[34,35]$, and were promptly adopted at our institution as a standard or care. In our study, whether mothers received perinatal antibiotics for prevention of GBS disease was not recorded. However, maternal factors (including the receipt of antenatal antibiotics or steroid therapy) do not seem to have any significant association with the development of late-onset sepsis [1,2], so the omission of these variables is likely to have had little, if any, influence on reported outcomes.

The reasons for the significantly higher proportion of LOS due to S. epidermidis during the second study period are unknown. Zaidi et al. [36] have suggested that the preponderance of coagulase-negative staphylococci in neonatal sepsis may be related to the adoption of more advanced tertiary neonatal care practices. This could be the case in our study. Whether perinatal administration of antibiotics for prevention of GBS disease plays any role is yet unclear, although it is unlikely. Whatever the reason, it may certainly have had the potential to influence on reported efficacy results. There is a number of evidences showing that neonates with coagulase-negative staphylococcal bacteremia are less likely to die than neonates infected with other pathogens, particularly Gramnegative rods $[1,2,37]$. Furthermore, shorter courses of antibiotics for sepsis due to $S$. epidermidis may be as efficacious as longer courses [5].

The non-randomized nature of our study and the limitations outlined above preclude any definite conclusions. However, we think that incorporating serum CRP serial determinations as a key decision-making criterion about duration of antibiotic treatment for primary culturepositive LOS may shorten exposure of neonates to antibiotics with no increase in adverse outcomes. The findings of this study warrant further evaluation in randomized trials.

\section{References}

1. Stoll B.J., Gordon T., Korones S., et al. Late-onset sepsis in very low birth weight neonates: a report from the National Institute of Child Health and Human Development Neonatal Reseach Network. J Pediatr 1996;129:63-71.

2. Stoll B.J., Hansen N., Fanaroff A.A., et al. Late-onset sepsis in very low birth weight neonates: the experience of the NICHD Neonatal Reseach Network. Pediatrics 2002;110:285-91.

3. Makhoul I.R., Sujov P., Smolkin T., et al. Epidemiological, clinical, and microbiological characteristics of late-onset sepsis among very low birth weight infants in Israel: a national survey. Pediatrics 2002;109:34-9.

4. Isaacs D., Barfield C., Clothier T., et al. Late-onset infections of infants in neonatal units. J Paediatr Child Health 1996;32:158-61.

5. Rubin L.G., Sánchez P.J., Siegel J., et al. Evaluation and treatment of neonates with suspected late-onset sepsis: a survey of neonatologists' practices. Pediatrics 2002;110:E42.

6. Yancey M.K., Duff P., Kubilis P., et al. Risk factors for neonatal sepsis. Obstet Gynecol 1996;87:188-94.

7. Isaacs D. Rationing antibiotic use in neonatal units. Arch Dis Child Fetal Neonatal Ed 2000;82:F1-F2.

8. Ehl S., Gering B., Bartmann P., et al. C-reactive protein in a useful marker for guiding duration of antibiotic therapy in suspected neonatal bacterial infection. Pediatrics 1997;99:216-21.

9. Adams-Chapman I., Stoll B.J. Adjunctive therapies for treatment or prevention of sepsis. Semin Pediatr Infect Dis 2001;12:211-9.

10. Ng P.C. Diagnostic markers of infection in neonates. Arch Dis Child Fetal Neonatal Ed 2004;89:229-35.

11. Garner J.S., the Hospital Infection Control Practices Advisory Committee. Guideline for isolation precautions in hospitals. Infect Control Hospital Epidemiol 1996;17:53-80.

12. Guidelines for prevention of nosocomial pneumonia. Centers for Disease Control and Prevention. MMWR Recomm Rep 1997;46(RR-1):1-79.

13. Pearson M.L. Guideline for prevention of intravascular devicerelated infections. Hospital Infection Control Practices Advisory Committee. Infect Control Hosp Epidemiol 1996;17:438-73.

14. Garner J.S., Jarvis W.R., Emori T.G., et al. CDC definitions for nosocomial infections, 1988. Am J Infect Control 1988; $16: 128-40$.

15. Goldmann D.A., Weinstein R.A., Wenzel R.P., et al. Strategies to prevent and control the emergence and spread of antimicrobialresistant microorganisms in hospitals. A challenge to hospital leadership. JAMA 1996;275:234-40.

16. Hengst J.M. The role of C-reactive protein in the evaluation and management of infants with suspected sepsis. Adv Neonatal Care 2003;3:3-13.

17. Steel D.M., Whitehead A.S. The major acute phase reactants: Creactive protein, serum amyloid $\mathrm{P}$ component and serum amyloid A protein. Immunol Today 1994;15;81-8.

18. Pepys M.B., Baltz M.L. Acute phase proteins with special reference to C-reactive protein and related proteins (pentaxins) and serum amyloid A protein. Adv Immunol 1983;34:141-212.

19. Ishibashi M., Takemura Y., Ishida H., et al. C-reactive protein kinetics in newborns: application of a high-sensitivity analytic method in its determination. Clin Chem 2002;48:1103-6.

20. Pepys M.B. C-reactive protein fifty years on. Lancet 1981;1:653-7.

21. Pourcyrous M., Bada H.S., Korones S.B., et al. Significance of serial C-reactive protein responses in neonatal infection and other disorders. Pediatrics 1993;92:431-5.

22. Gabay C., Kushner I. Acute-phase proteins and other systemic responses to inflammation. N Engl J Med 1993;340:448-54.

23. Benitz W.E., Han M.Y., Madan A., Ramachandra P. Serial serum C-reactive protein levels in the diagnosis of neonatal infection. Pediatrics 1998;102:E41. 
24. Ehl S., Gering B., Pohlandt F. A detailed analysis of changes in serum C-reactive protein levels in neonates treated for bacterial infection. Eur J Pediatr 1999;158:238-42.

25. Young B., Gleeson M., Cripps A.W. C-reactive protein: a critical review. Pathology 1991;23:118-24.

26. Franz A.R, Steinbach G., Kron M., Pohlandt F. Reduction of unnecessary antibiotic therapy in newborn infants using interleukin-8 and C-reactive protein as markers of bacterial infections. Pediatrics 1999;104:447-53.

27. Kawamura M., Nishida $H$. The usefulness of serial C-reactive protein measurement in managing neonatal infection. Acta Paediatr 1995;84:10-3.

28. Philip A.G., Mills P.C. Use of C-reactive protein in minimizing antibiotic exposure: experience with infants initially admitted to a well-baby nursery. Pediatrics 2000;106:401-11.

29. Russell G.A., Smyth A., Cooke R.W. Receiver operating characteristic curves for comparison of serial neutrophil band forms and C-reactive protein in neonates at risk for infection. Arch Dis Child 1992;67:808-12.

30. Chiesa C., Panero A., Osborn J.F., et al. Diagnosis of neonatal sepsis: a clinical and laboratory challenge. Clin Chem 2004;50:279-87.
31. Franz A.R., Bauer K., Schalk A., et al. Measurement of interleukin 8 in combination with C-reactive protein reduced unnecessary antibiotic therapy in newborn infants: a multicecenter, randomized, controlled trial. Pediatrics 2004;114:1-8.

32. Jaye D.L., Waites K.B. Clinical applications of C-reactive protein in pediatrics. Pediatr Infect Dis J 1997;16:735-46.

33. Stoll B.J., Hansen N., Fanaroff A.A., et al. Changes in pathogens causing early-onset sepsis in very-low-birth-weight infants. $\mathrm{N}$ Engl J Med 2002;347:240-7

34. American College of Obstetricians and Gynecologists, Committee on Obstetric Practice. Prevention of early-onset group B streptococcal disease in newborns [Opinion 173]. Washington, D.C.: American College of Obstetricians and Gynecologists, 1996.

35. CDC. Prevention of perinatal group B streptococcal disease: a public health perspective. MMWR 1996;45(RR-7):1-24.

36. Zaidi A.K., Huskins W.C., Thaver D., et al. Hospital-acquired neonatal infections in developing countries. Lancet 2005;365:1175-88.

37. Gray J.E., Richardson D.K., McCormick M.C., Goldmann D.A. Coagulase-negative staphylococcal bacteremia among very low birth weight infants: relation to admission illness severity, resource use, and outcome. Pediatrics 1995;95:225-30. 